\title{
Aproximación del teletrabajo en el ámbito universitario
}

\section{Approach to teleworking in the university environment}

\author{
Brito-Cruz Teresa del Jesús ${ }^{1}$, Lara-Gamboa Carmen Cecilia², Morales-Diego Alicia Mariela², Sánchez-May María del Carmen³, \\ Perez-Jaimes Alejandra Karina ${ }^{4}$ y Torres-Zapata Ángel Esteban ${ }^{1 *}$
}

1 Universidad Autónoma del Carmen.

2 Universidad Autónoma de Campeche.

3 Universidad Juárez Autónoma de Tabasco.

4 Universidad Autónoma del Estado de México.

*Autor para la correspondencia: Torres-Zapata Ángel esteban.etorre@pampano.unacar.mx

\section{Resumen}

El Teletrabajo hoy en día ha adquirido mayor visibilidad en la aplicabilidad en los contextos organizacionales, incluyendo el ámbito educativo, trabajar virtualmente o a distancia es una nueva manera de trabajar y los beneficios de la aplicabilidad en las instituciones de educación superior en México cada vez poseen mayor alcance. El objetivo del presente estudio de investigación fue describir el estado actual del conocimiento del Teletrabajo en el ámbito universitario. Es un estudio documental descriptivo, mediante la revisión de artículos científicos utilizando el enunciado clave en español: "Teletrabajo en el ámbito universitario" en el aparato de búsqueda Google Académico. Los datos se registraron en una matriz de doble entrada, identificando la correspondencia entre objetivos, metodología, resultados y conclusiones. Los resultados de la revisión muestran suficiente evidencia para recomendar continuar el abordaje de este tema.

Palabras clave: Teletrabajo, educación, universidad.

\section{Abstract}

Nowadays, teleworking applications have become a common sight in organizational contexts, including the education field, working at home or within a virtual environment is a new way of working, benefits associated with its applications on Mexico's higher education institutions have extended their reach. The main goal of this research was to describe the current state of knowledge of teleworking in the university's environment. This is descriptive bibliographical research; Scholar Google was employed to look for scientific articles with the sentence: "Teleworking in the university environment". Entries were recorded on a double-entry matrix identifying a correspondence between objectives, method, results, and conclusions. Results obtained from research throw enough evidence to pursue further study.

Key words: Teleworking, education, university.

DOI 10.46588/invurnus.v16i1.43

Recibido 20/06/2021

Aceptado 9/07/2021

Publicado 15/07/2021 


\section{Introducción}

En el 2020, la humanidad ha sido azotada por un fenómeno inesperado denominado coronavirus SARSCoV2 (COVID-19), este tipo de fenómenos se caracterizan por ser impredecibles, se trata de uno de tantos fenómenos aleatorios cargados de azar e incerteza, susceptibles de presentarse tanto en la naturaleza como en la sociedad; para su paulatina comprensión, se ha de acudir a los modelos epidemiológicos focalizados en la estimación de ciertas probabilidades y al manejo de diversos tipos de variables asociadas con esta clase de fenómenos pandémicos (Escudero et al., 2020).

De manera general, la pandemia causada por la COVID-19, implicó la emisión de políticas públicas emergentes universales y particulares de cada estado para mantener el equilibrio en la sociedad al tiempo que se atendía la contingencia, la cual implicaba de forma inmediata y contundente el resguardo sanitario, adoptando los protocolos "quédate en casa" y "sana distancia" para con ello disminuir los índices de infestación por COVID-19 (Lozano-Díaz et. al., 2020). En este contexto, el gobierno mexicano emanó una serie de acuerdos para atender la emergencia sanitaria causada por la COVID-19, los cuales fueron expedidos para ser aplicados a partir del 16 de marzo de 2020; desde esta fecha, se realizan medidas y acciones que les procurarán autocuidado y autoprotección ante la emergencia sanitaria al evitar actos que involucren o conlleven a aglomeraciones de personas para así evitar la propagación del contagio a lo largo y ancho del territorio nacional, denominado "quédate en casa" y "sana distancia" (Navarrete, Manzanilla, y Ocaña, 2020). Tal medida ha implicado que millones de trabajadores y estudiantes entren en periodos largos de confinamiento en sus hogares, hasta aprovisionar los hospitales y obtener información adicional para ofrecer un manejo pertinente de la pandemia; este confinamiento, generó una parálisis temporal del sistema productivo en el mundo, el cierre de los aeropuertos y los diversos medios de trasporte, y la búsqueda de alternativas para que el sistema educativo en sus diferentes niveles siguiera funcionando desde los hogares, con la ayuda de los medios virtuales, ya que el ciclo escolar estaba al 50\% de su ejecución, por lo que debía realizarse una adecuación curricular que permitiera, salvaguardar la integridad física y de salud de la comunidad académica (administrativos, docentes, directivos y estudiantes), y también, dar continuidad al proceso formativo y concluir el ciclo escolar sin más contratiempos (Vergara et. al., 2020).

El sistema educativo no estaba del todo preparado para afrontar estas eventualidades caracterizadas por la presencia de riesgo, incertidumbre y azar en la vida de los estudiantes, docentes y padres de familia (Pantoja, Valdivieso, y Burbano, 2020), ya que, un alto porcentaje de las instituciones educativas apenas estaba haciendo inmersiones en el uso de las tecnologías de la información y las comunicaciones (TIC) para mejorar los procesos de enseñanza y aprendizaje implícitos en el currículo escolar o para dinamizar los planes de estudios a nivel universitario con diversas metodologías (Barzola-López, Suárez-Véliz y Arcos-Coba, 2020). Es pertinente reconocer que, la educación superior pública no estaba preparada para afrontar una situación intempestiva como la ocasionada por la COVID-19; las decisiones rectorales de llamar a confinamiento a los diferentes niveles universitarios, a fin de preservar la vida y disminuir el contagio, llevaron a la búsqueda de soluciones en metodologías, pedagogías y didácticas propias de la educación a distancia y virtual (Poveda-Pineda y Cifuentes-Medina, 2020).

Esta situación de salud lo ha cambiado todo y ha instado a los docentes universitarios a transitar y adaptarse rápidamente a una variada gama de eventos inesperados, entre ellos, la incursión en el manejo de las TIC's como un recurso indispensable para desarrollar sus actividades universitarias o el uso eficiente de sus competencias docentes en ámbitos virtuales, tales cambios han generado diversos tipos de afectaciones en la vida cotidiana del docente universitario, tanto a nivel físico y psicológico como económico y social; no 
obstante, el maestro ha tenido que acudir a su capacidad de resiliencia para superar estas circunstancias difíciles y sacar adelante sus actividades de academia, investigación y proyección social (Figallo, González, y Diestra, 2020).

Por tal motivo, el presente trabajo tiene el objetivo de caracterizar el estado actual del teletrabajo en el ámbito universitario en México, como una alternativa ante contingencia de la COVID-19.

\section{Metodología}

La investigación fue un estudio documental descriptivo; se tuvo como muestra, los documentos científicos en los que se cuenta con un abordaje en materia de investigación en contextos educativos relacionados con la aplicabilidad del Teletrabajo, siguiendo la metodología de Torres et. al., 2018.

Se empleó el buscador Google Académico para el rastreo de información, utilizando el enunciado "Teletrabajo en el ámbito Universitario", en idioma en español, durante el periodo de un mes (abril-mayo 2021), donde se incluyeron los trabajos bajo las siguientes consideraciones:

1. Se consideró el objeto del estudio (Teletrabajo en el contexto universitario) y su delimitación geográfica (México).

2. Investigaciones con enfoques cuantitativos, cualitativos y mixtos

3. Artículos a partir de enero 2012 a febrero 2021.

Los trabajos excluidos fueron aquellos que no cumplieron con los criterios antes descritos o que el contenido temático duplicaba la información. La búsqueda de información se realizó por los integrantes del grupo disciplinar "Nutrición, Educación y Administración" de forma independiente. Los datos fueron ingresados en una hoja de cálculo de Excel diseñada para el estudio.

La selección de los documentos se basó en una exhaustiva revisión de títulos, resúmenes y palabras claves de los estudios. De no encontrarse la información necesaria en el resumen publicado, se buscaba el artículo completo. La calidad metodológica y científica de los artículos considerados en este trabajo, fue determinada al ser publicados en revistas arbitradas e indexadas, dado los criterios científicos a los que deben apegarse y las revisiones por expertos a las que son sujetos.

La lectura crítica a texto completo de los documentos científicos que cumplieron los supuestos de inclusión definidos permitió la categorización como: I) Cumple la totalidad de los criterios de inclusión, existe bajo riesgo de sesgo; II) Uno o más criterios se cumplen solo parcialmente, existe moderado riesgo de sesgo y III) Uno o más criterios no se cumplen, existe alto riesgo de sesgo.

La variabilidad, fiabilidad y validez, se estableció a través del diseño y uso de una matriz de doble entrada, que permitió identificar la correspondencia entre los objetivos, la metodología, los resultados y las conclusiones entre los artículos.

\section{Resultados}

Se utilizó el aparato de búsqueda "Google Académico", versión en castellano de "Google Scholar", con perfil académico y científico, ya que rastrea todo tipo de documentación científico-académica localizable en 
la Web, tanto referencias bibliográficas como documentos íntegros; indexados, editoriales, bibliotecas, repositorios, bases de datos bibliográficas, entre otros. Entre los resultados que genera, se pueden encontrar citas, enlaces a libros, artículos de revistas científicas, comunicaciones y ponencias en congresos, informes científico-técnicos, tesis, tesinas y archivos depositados en repositorios. Esta herramienta, es utilizada frecuentemente por estudiantes universitarios e investigadores, para elaborar documentos científicos y académicos de diversas áreas (Torres-Zapata et. al., 2018).

Al ingresar en el buscador el enunciado "Teletrabajo en el ámbito universitario", se generaron 10,200 resultados en 0.03 segundos, de los cuales, el $0.78 \%$ cumplen con los criterios de inclusión; y con el enunciado. Las características de los estudios incluidos se presentan en la tabla 1 y tabla 2.

El $0.78 \%$ de los documentos científicos fueron finalmente incluidos en la revisión, el $12.5 \%$ de los estudios poseen enfoques mixtos (cuantitativos y cualitativos) (Ruiz y Aguirre, 2020), y el 87.5\% son estudios con enfoque cualitativo (Navarrete, Manzanilla y Ocaña, 2020; Chávez, Martínez y Dávila, 2020; Jiménez, 2012; Prince, 2020; Ordorika, 2020; Villela y Contreras, 2021). Los resultados nos permiten visibilizar que actualmente no se posee un abordaje amplio del tema del teletrabajo en los contextos universitarios o por lo menos dichos procesos no están documentados a través de la investigación gestada dentro de la comunidad del conocimiento.

\section{Discusión}

La Organización Internacional del Trabajo define al teletrabajo como un nuevo esquema de laborar, en un lugar alejado de una oficina o de las instalaciones de la Institución u organización a la que pertenecen los colaboradores, separando con ello al trabajador del contacto personal con colegas, así como con quienes los lideran. Siendo así en un contexto amplio la ejecución del trabajo a distancia prestado mediante el uso de las telecomunicaciones y de las TIC’s (Navarrete, Manzanilla y Ocaña, 2020; Chávez, Martínez y Dávila,2020). El teletrabajo es una actividad que moderniza la forma en que actualmente laboramos y tiene impacto en todos los contextos, incluido el ámbito universitario (Chávez, Martínez y Dávila,2020).

Cabe destacar que a nivel mundial se han experimentado cambios en los diversos ámbitos de la sociedad, específicamente en el universitario se ha vislumbrado un impacto fuerte y directo sobre el rendimiento académico y los medios en los que se imparte la enseñanza-aprendizaje en nuestro país. (Figallo, González, y Diestra, 2020). En el ámbito de la educación no cabe duda de que la integración de las TIC's a las instituciones educativas, ha generado un aumento de la transferencia de los aprendizajes a la sociedad mediante una nueva modalidad. Es evidente el hecho de que en el último año el teletrabajo ha adquirido mayor visibilidad esto a partir del confinamiento, siendo el parteaguas para decir que el ejercicio del teletrabajo es una realidad que va en aumento en su aplicabilidad en las Instituciones de educación superior en nuestro país, aun cuando conforme a algunas de las investigaciones llevadas a cabo en México con base a la percepción del teletrabajo por parte de los docentes y universitarios en donde refieren que poseen la disposición y disponibilidad para adaptarse a esta nueva modalidad de formación profesional, dejando entrever la necesidad que poseen, en relación a formarse, capacitarse, adecuarse y adaptarse a la aplicación de las TIC’s en pro de la educación (Jiménez, 2012). 
Tabla 1. Características generales de los documentos analizados. Parte I.

\begin{tabular}{|c|c|}
\hline \multicolumn{2}{|r|}{ Jiménez, 2012} \\
\hline Título & $\begin{array}{l}\text { La tele docente universitario, tipo especial de teletrabajo que coadyuva al desarrollo } \\
\text { de los sistemas de educación no convencionales }\end{array}$ \\
\hline Objetivo & Establecer el estado del arte del teletrabajo \\
\hline Metodología & Estudio documental descriptivo \\
\hline Resultado & Incorporar en México la figura jurídica del teletrabajador en la Ley Federal del Trabajo \\
\hline Conclusiones & $\begin{array}{l}\text { El teletrabajo en México no está regulado, se propone por ello incorporar esta figura } \\
\text { de modo general en la Ley Federal del Trabajo }\end{array}$ \\
\hline \multicolumn{2}{|r|}{ Ordorika, 2020} \\
\hline Título & Pandemia y educación superior \\
\hline Objetivo & Describir el impacto de la pandemia en la educación superior. Utilidad del teletrabajo \\
\hline Metodología & Revisión documental \\
\hline Resultados & $\begin{array}{l}\text { En este contexto, en los próximos meses y años es muy probable que se den cambios } \\
\text { relevantes en las formas tradicionales de trabajo académico y en la organización de las } \\
\text { IES }\end{array}$ \\
\hline Conclusiones & $\begin{array}{l}\text { En México es claro que será necesario establecer una nueva agenda de } \\
\text { transformaciones para las IES en general y para cada una de ellas a partir de sus } \\
\text { particularidades }\end{array}$ \\
\hline \multicolumn{2}{|r|}{ Chávez, Martínez y Dávila, 2020 } \\
\hline Título & Educación a Distancia y Teletrabajo \\
\hline Objetivo & $\begin{array}{l}\text { Medir percepción sobre la importancia } \\
\text { del trabajo a distancia }\end{array}$ \\
\hline Metodología & Investigación con enfoque cualitativo \\
\hline Resultados & $\begin{array}{l}\text { El } 100 \% \text { considera necesaria la educación a distancia, el } 90 \% \text { considera que la } \\
\text { educación a distancia debe ofertarse en todos los niveles educativos, sólo el } 10 \% \\
\text { considera que no }\end{array}$ \\
\hline Conclusiones & El 100\% considera que el teletrabajo debe ser una opción permanente para trabajar \\
\hline \multicolumn{2}{|r|}{ Toscano, Contreras-Castillo y Barón Ramírez, 2020} \\
\hline Título & $\begin{array}{l}\text { Una Reflexión al Respecto de la Pandemia por COVID-19. La Educación Superior y } \\
\text { las Acciones Emprendidas para el Regreso a la Nueva Normalidad }\end{array}$ \\
\hline Objetivo & $\begin{array}{l}\text { Analizar el impacto de la pandemia por el virus COVID-19 con énfasis en la Educación } \\
\text { Superior y las acciones académicas y administrativas emprendidas durante la } \\
\text { pandemia. Analizar el impacto de la pandemia por el virus COVID-19 con énfasis en } \\
\text { la Educación Superior y las acciones académicas y administrativas emprendidas } \\
\text { durante la pandemia }\end{array}$ \\
\hline Metodología & Ensayo \\
\hline Resultados & $\begin{array}{l}\text { En México como en todo América Latina, la Pandemia por COVID-19 no solo pone } \\
\text { en descubierto las desigualdades existentes en el país, sino que desnuda la precariedad } \\
\text { del contexto laboral, del sistema de salud y de su sistema educativo }\end{array}$ \\
\hline Conclusiones & $\begin{array}{l}\text { La Educación no es un ente aislado, en las IES se trabaja con seres sociales, personas } \\
\text { que están viviendo todos estos claroscuros de la pandemia por COVID-19, por tanto, } \\
\text { reconstruir una nueva normalidad para la educación superior sin considerar todos estos } \\
\text { aspectos, sería una falacia }\end{array}$ \\
\hline
\end{tabular}


Tabla 2. Características generales de los documentos analizados. Parte 2.

\begin{tabular}{|c|c|}
\hline \multicolumn{2}{|r|}{ Walker, 2020} \\
\hline Título & $\begin{array}{l}\text { Tendencias en el campo de la educación superior y su incidencia en el Trabajo Docente } \\
\text { Universitario }\end{array}$ \\
\hline Objetivo & Analizar las tendencias en el teletrabajo en el ámbito educativo \\
\hline Metodología & Estudio documental descriptivo. \\
\hline Resultados & $\begin{array}{l}\text { El aumento del teletrabajo y las formas virtuales de vinculación van atomizando al } \\
\text { colectivo docente de modo que se dedique "cada cual a lo suyo" }\end{array}$ \\
\hline Conclusiones & $\begin{array}{l}\text { Las tendencias que se dirigen a la fragmentación, la estratificación y la } \\
\text { individualización del profesorado universitario logran consolidarse por la connivencia } \\
\text { de los propios docentes que desarrollan estrategias adaptativas para preservar o mejorar } \\
\text { su posición en el campo universitario }\end{array}$ \\
\hline \multicolumn{2}{|r|}{ Ruíz y Aguirre, 2020} \\
\hline Título & $\begin{array}{l}\text { Tecnologías de la información y comunicación y docencia: dimensiones culturales, } \\
\text { sociales y pedagogía }\end{array}$ \\
\hline Objetivo: & $\begin{array}{l}\text { Indaga sobre cómo vivieron cinco docentes y cinco coordinadores académicos que } \\
\text { participan en programas de educación a distancia en Educación Superior, el uso y } \\
\text { apropiación de las TIC para generar ambientes de aprendizaje virtuales }\end{array}$ \\
\hline Metodología & La investigación es mixta, con apoya del método etnográfico virtual \\
\hline Resultados & $\begin{array}{l}\text { Los docentes experimentan cambios socioculturales, disciplinares y pedagógicos } \\
\text { cuando a su práctica se insertan las TIC. Ahora no sólo basta tener conocimiento en la } \\
\text { disciplina, sino otros saberes que apoyan los procesos de comunicación, interacción y } \\
\text { de motivación que se dan en diversos ambientes de aprendizaje }\end{array}$ \\
\hline \multicolumn{2}{|r|}{ Villela y Contreras, 2021} \\
\hline Título & $\begin{array}{l}\text { La brecha digital como una nueva capa de vulnerabilidad que afecta el acceso a la } \\
\text { educación en México }\end{array}$ \\
\hline Objetivo & $\begin{array}{l}\text { Visibilizar aquellas áreas que requieren atención y permitirían tomar acciones positivas } \\
\text { para reducir las capas de vulnerabilidad que devienen de la brecha digital }\end{array}$ \\
\hline Metodología & Análisis ensayístico \\
\hline Resultados & $\begin{array}{l}\text { Las tic's para teletrabajo y teleeducación, lo más probable es que muchos de estos } \\
\text { cambios hayan llegado para quedarse }\end{array}$ \\
\hline Conclusiones & $\begin{array}{l}\text { Se debe trabajar en los cambios a las leyes de trabajo que tengan en cuenta el teletrabajo } \\
\text { o trabajo desde casa, capacitar a los padres, las madres o los tutores en materia docente, } \\
\text { entre otros }\end{array}$ \\
\hline \multicolumn{2}{|r|}{ Prince, 2021} \\
\hline Título & $\begin{array}{l}\text { La brecha digital como obstáculo al derecho universal a la educación en tiempos de } \\
\text { pandemia }\end{array}$ \\
\hline Objetivo & $\begin{array}{l}\text { Analizar las consecuencias de la brecha digital como un obstáculo al acceso sobre el } \\
\text { derecho universal a la educación en tiempos de pandemia }\end{array}$ \\
\hline Metodología & Estudio de enfoque cualitativo y diseño documental con análisis de textos \\
\hline Resultados & $\begin{array}{l}\text { La brecha digital ha condicionado el ejercicio del derecho a la educación e igualmente } \\
\text { ahonda esas diferencias porque de acuerdo con las oportunidades que tenga cada } \\
\text { individuo }\end{array}$ \\
\hline Conclusiones & $\begin{array}{l}\text { La pandemia impulso el inicio del cambio de paradigmas en la realización del acto } \\
\text { educativo para que se ajuste a los nuevos tiempos }\end{array}$ \\
\hline
\end{tabular}


La presente revisión muestra que los docentes actualmente experimentan cambios socioculturales, disciplinares y pedagógicos en cuanto a su práctica en relación con la incorporación de las TIC's (Ruiz y Aguirre, 2020), dando inicio con ello al cambio de paradigmas que dan pauta a los procesos de comunicación, interacción y de motivación que se dan en diversos ambientes de aprendizaje en el país. La información plantea finalmente que la revisión documental precisa una baja frecuencia en el establecimiento de esta línea de investigación en México, resaltando la importancia de la necesidad de establecer futuras investigaciones en el marco de este tema, ya que el teletrabajo en un sentido amplio es considerado un nuevo esquema de trabajo que llegó para implementarse y establecerse en los diversos contextos organizacionales haciendo hincapié a un área de oportunidad para la sociedad misma.

Uno de los puntos que es necesario resaltar es la existencia de la brecha digital que no se había abordado con diferentes enfoques, ya que cuando se hace referencia a la brecha digital, también se realiza un llamado al concepto de desigualdad, pues en parte versa sobre los trances que vive parte de la población mundial para acceder a los servicios de conexión por intermedio de las TIC's (Prince, 2021).

Finalmente, es importante resaltar la necesidad de futuras investigaciones, con aplicaciones en entornos diversos, desde el abordaje de los factores psicosociales de riesgo ya que es un sistema de trabajo que vino para quedarse como una modalidad de educación híbrida, por tanto, es importante evaluar el impacto del aprendizaje y generar estrategias de capacitación para el personal administrativo, docente y los estudiantes universitarios.

\section{Conclusiones}

La situación de salud que generó la COVID-19 impulsó el inicio del cambio de paradigma en las instituciones de educación superior en México, las cuales deberán adaptarse a una educación híbrida, generando con ello la necesidad de implementar cambios en la legislación del trabajo en donde se tome en consideración el teletrabajo en el ámbito universitario con todas las implicaciones que ello conlleva, para regular el ejercicio profesional mediante esta nueva modalidad de trabajo y lograr una educación de calidad, ya que el personal administrativo, docente y estudiantes experimentan cambios socioculturales, disciplinares y pedagógicos dónde si bien las TIC's ya estaban implementadas y formaban parte de la malla curricular es evidente que hoy en día se requiere que se fortalezcan conforme a los requerimientos de la nueva complejidad de la demanda actual.

\section{Referencias}

Barzola-López, L., Suárez-Veliz, M. y Arcos-Coba, J. (2020). La influencia de las TIC’s en el desarrollo académico de los estudiantes universitarios en tiempos de pandemia por COVID-19. Dominio de las Ciencias, 6(4), 370386. doi:http://dx.doi.org/10.23857/dc.v6i4.1473

Chávez, J., Martínez, J. y Dávila, R. (2020). Educación a distancia y teletrabajo. Daena: International Journal of Good Conscience, 15(1), 264-277. Recuperado de http://www.spentamexico.org/v15-n1/A19.15(1)264-277.pdf

Escudero, X., Guarner, J., Galindo-Fraga, A., Escudero-Salamanca, M., Alcocer-Gamba, M. A. y Río, C. (2020). La pandemia de coronavirus SARS-CoV-2 (COVID-19): Situación actual e implicaciones para México. Archivos de cardiología de México, 90 (Supl. 1), 7-14. https://doi.org/10.24875/acm.m20000064

Figallo, F., González, M. y Diestra, V. (2020). Perú: Educación superior en el contexto de la pandemia POR el COVID19. Revista de Educación Superior en América Latina, (8), 20-28. doi:10.14482/esal.8.378.85 
Jiménez, A. (2012). La tele docente universitario, tipo especial de teletrabajo que coadyuva al desarrollo de los sistemas de educación no convencionales. Paper presented at Teleword Costa Rica 2012, Costa Rica. Recuperado de https://cutt.ly/qnNjluM

Lozano-Díaz, A., Fernández-Prados, J., Figueredo, V. y Martínez, A. (2020). Impactos del confinamiento por el COVID-19 entre universitarios: Satisfacción vital, Resiliencia Y capital social online. International Journal of Sociology of Education, 79. doi:10.17583/rise.2020.5925

Navarrete, Z., Manzanilla, H. y Ocaña, L. (2020). Políticas implementadas por el gobierno mexicano frente al COVID19. El Caso de la educación básica. Revista Latinoamericana de Estudios Educativos, 50(ESPECIAL), 143-172. doi: 10.48102/rlee.2020.50.especial.100

Ordorika, I. (2020). Pandemia y educación superior. Revista de la educación superior, 49(194), 18.https://doi.org/10.36857/resu.2020.194.1120

Pantoja, V., Valdivieso, M. y Burbano, A. (2020). Teletrabajo académico afectado POR el coronavirus: Una mirada desde UN grupo focal de profesores universitarios. Espacios, 41(42), 335-348. doi:10.48082/espaciosa20v41n42p29

Poveda-Pineda, D. y Cifuentes-Medina, E. (2020). Incorporación de las tecnologías de información y comunicación (TIC) durante el proceso de aprendizaje en la educación superior. Formación universitaria, 13(6), 95-104. https://dx.doi.org/10.4067/S0718-50062020000600095

Prince, A. (2021). La brecha digital como obstáculo al derecho universal a la educación en tiempos de pandemia. Journal of the Academy, (4), 26-41. Recuperado de https://journalacademy.net/index.php/revista/article/view/44/32

Ruiz, M. y Aguirre, G. (2020). Tecnologías de la información y comunicación y docencia: dimensiones culturales, sociales y pedagógicas. Revista de Transformación Educativa, 273-299. Recuperado de https://cutt.ly/XnNk1DH

Torres-Zapata, A., Zapata-Gerónimo, D., Rivera-Domínguez, J. y Acuña-Lara, J. (2018). El obeso de peso normal. RESPYN Revista de Salud Pública y Nutrición, 17(2), 24-31. doi:10.29105/respyn17.2-4

Toscano, B., Contreras, J. y Barón, N. (2020). Una Reflexión al Respecto de la Pandemia por COVID-19. La Educación Superior y las Acciones Emprendidas para el Regreso a la Nueva Normalidad. Tecnología Educativa, 7(2), 66-83. Recuperado de https://terc.mx/index.php/terc/article/view/7

Vergara, E., Vergara, R., Álvarez, Ma., Camacho, L. y Gálvez, J. (2020). Educación médica a distancia en tiempos de COVID-19. Educación Médica Superior, 34(2), e2383. Epub 01 de junio de 2020. Recuperado de http://scielo.sld.cu/scielo.php?script=sci_arttext\&pid=S0864-21412020000200025\&lng=es\&tlng=.

Villela, F. y Contreras, D. (2021). La brecha digital como una nueva capa de vulnerabilidad que afecta el acceso a la educación en México. Academia y Virtualidad, 14(1), 183-187. Recuperado de https://revistas.unimilitar.edu.co/index.php/ravi/article/view/5395

Walker, V. (2020). Tendencias en el campo de la educación superior y su incidencia en el Trabajo Docente Universitario. Revista de la educación superior, 49(193), 107-127.https://doi.org/10.3 